\title{
Simulation of active Brownian particles in optical potentials
}

Giorgio Volpe Sylvain Gigan Giovanni Volpe 


\title{
Simulation of Active Brownian Particles in Optical Potentials
}

\author{
Giorgio Volpe $^{* a}$, Sylvain Gigan ${ }^{\mathrm{a}}$, Giovanni Volpe ${ }^{\mathrm{b}}$ \\ ${ }^{a}$ Laboratoire Kastler Brossel, UMR8552 of CNRS and Université Pierre et Marie Curie, Ecole \\ Normale Supérieure and Collège de France, 24 rue Lhomond, 75005 Paris, France; \\ ${ }^{\mathrm{b}}$ Physics Department, Bilkent University, Cankaya, 06800 Ankara, Turkey
}

\begin{abstract}
Optical forces can affect the motion of a Brownian particle. For example, optical tweezers use optical forces to trap a particle at a desirable position. Unlike passive Brownian particles, active Brownian particles, also known as microswimmers, propel themselves with directed motion and thus drive themselves out of equilibrium. Understanding their motion in a confined potential can provide insight into out-of-equilibrium phenomena associated with biological examples such as bacteria, as well as with artificial microswimmers. We discuss how to mathematically model their motion in an optical potential using a set of stochastic differential equations and how to numerically simulate it using the corresponding set of finite difference equations.
\end{abstract}

Keywords: optical forces, Brownian motion, stochastic differential equations, numerical simulations, active Brownian particles, microswimmers

\section{INTRODUCTION}

We present a simple algorithm to simulate an active Brownian particle, also known as microswimmer, in a twodimensional field of optical forces [1], as discussed in more detail in Ref. [2-3]. We provide an implementation of this algorithm using $\mathrm{MatLab}^{\circledR}$, because this language is widely employed in science and engineering. All algorithms can also be translated straightforwardly in the freeware programming languages SciLab [4] or Octave [5].

In first place, it is useful to simulate the motion of an active Brownian particle in a field of optical forces to gain insight into out of equilibrium physics and the interaction of active particles with physical potentials. Active Brownian motion, in fact, has attracted a lot of interest from the biology and physics communities [6-7]: several types of microscopic biological entities perform active Brownian motion and artificial active particles can be used to localize, pick up, and deliver nanoscopic objects, e.g., in bioremediation, drug delivery, and gene therapy [8-15]. While the motion of passive Brownian particles is driven by equilibrium thermal fluctuations, active Brownian particles are able to propel themselves, exhibiting an interplay between random fluctuations and active swimming that drives them into an out-ofequilibrium status [16-17]. Such artificial active Brownian particles propel themselves by several mechanisms, such as by a periodic deformation of their shape or by phoresis in, e.g., an electric field or a chemotactic or temperature gradient [18-28]. Studying the motion of these active particles in an optical potential can help understand basic principles of their interaction with surface and physical barriers to optimize delivery applications.

\section{THEORETICAL MODEL}

In a bidimensional homogeneous environment, the motion of an active particle can be modeled as the combined action of three different processes [29-30]: a random diffusion process, an internal self-propelling force and, in the case of chiral active particles, a torque. In particular, the position $[\mathrm{x}(\mathrm{t}), \mathrm{y}(\mathrm{t})]$ of a spherical particle with radius $\mathrm{R}$ undergoes Brownian diffusion with translational diffusion coefficient:

$$
D_{T}=\frac{k_{B} T}{6 \pi \eta R}
$$

"'giorgio.volpe@1kb.ens.fr

Optical Trapping and Optical Micromanipulation XI, edited by Kishan Dholakia,

Gabriel C. Spalding, Proc. of SPIE Vol. 9164, 91642S · (C) 2014 SPIE

CCC code: $0277-786 X / 14 / \$ 18 \cdot$ doi: $10.1117 / 12.2061049$ 
where $\mathrm{k}_{\mathrm{B}}$ is the Boltzmann constant, $\mathrm{T}$ the temperature and $\eta$ the fluid viscosity. The particle self-propulsion results in a directed component of the motion, whose speed $\mathrm{v}$ we will assume to be constant and whose direction depends on the particle orientation $\varphi(t)$, as illustrated in Figure 1(a). Finally, $\varphi(t)$ undergoes rotational diffusion with rotational diffusion coefficient:

$$
D_{R}=\frac{k_{B} T}{8 \pi \eta R^{3}}
$$

For chiral active particles, $\varphi(t)$ also rotates with angular velocity $\Omega$ as a consequence of the torque acting on the particle [29-30], as shown in Figure 1(b). The sign of $\Omega$ determines the chirality of the particles. In the most general case, the motion of an active Brownian particle in a generic force field can be described by the following set of Langevin equations in two dimensions [2-3]:

$$
\left\{\begin{array}{c}
\varphi(t)=\Omega+\sqrt{2 D_{R}} W_{\varphi} \\
\dot{x}(t)=v \sin (\varphi(t))+\sqrt{2 D_{T}} W_{x}+F_{x}(t) \\
\dot{y}(t)=v \cos (\varphi(t))+\sqrt{2 D_{T}} W_{y}+F_{y}(t)
\end{array}\right.
$$

where $\mathrm{W}_{\varphi}, \mathrm{W}_{\mathrm{x}}$ and $\mathrm{W}_{\mathrm{y}}$ are independent white noise terms. Inertial effects are neglected because of the low Reynolds number regime [31] and $\left[F_{x}(x(t), t), F_{y}(y(t), t)\right]$ is an optical force acting on the particle that can vary both in space and time.

(a)

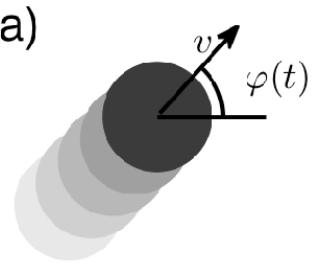

(b)

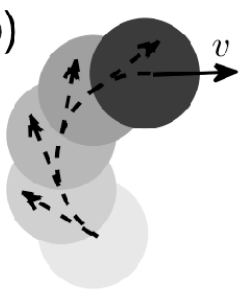

Figure 1. Active Brownian particles in two dimensions. (a) An active Brownian particle placed at $[\mathrm{x}(\mathrm{t})$, $\mathrm{y}(\mathrm{t})]$ is characterized by an orientation $\varphi(t)$ along which it propels itself with speed $v$ while it undergoes Brownian motion in both its position and orientation. (b) A chiral active Brownian particle also has a deterministic angular velocity $\Omega$ that, if the par- ticle's speed $\mathrm{v}>$ 0 , translates into a rotation around an effective external axis.

The continuous-time solution $[\varphi(t), x(t), y(t)]$ to the previous set of equations is approximated by the discrete-time sequence $\left[\varphi_{i}, x_{i}, y_{i}\right]$, which is the solution of the corresponding set of finite difference equations evaluated at regular time steps $\mathrm{t}_{\mathrm{i}}=\mathrm{i} \Delta \mathrm{t}$. If $\Delta \mathrm{t}$ is sufficiently small, $\left[\varphi_{\mathrm{i}}, \mathrm{x}_{\mathrm{i}}, \mathrm{y}_{\mathrm{i}}\right]=\left[\varphi\left(\mathrm{t}_{\mathrm{i}}\right), \mathrm{x}\left(\mathrm{t}_{\mathrm{i}}\right), \mathrm{y}\left(\mathrm{t}_{\mathrm{i}}\right)\right]$ following the procedure explained in Ref. [2-3].

\section{SIMULATION CODE}

In this section we present the MatLab ${ }^{\circledR}$ code that implements Eq. (1) following the codes provided with Ref. [2-3]. This function implements Eq. (1) for the case of a monostable optical trap. Inputs: number of samples N, timestep Dt, initial position $\mathrm{x} 1$, particle radius $\mathrm{R}$, temperature $\mathrm{T}$, fluid viscosity eta, particle speed $\mathrm{V}$, particle's angular velocity $\mathrm{W}$ and trap stiffness $\mathrm{k}$. Outputs: particle position $\mathrm{x}$ in meters and time $t$ in seconds.

function $[\mathrm{x}, \mathrm{t}]=$ ldtrapped_monostable $(\mathrm{N}, \mathrm{Dt}, \mathrm{x} 1, \mathrm{R}, \mathrm{T}$, eta, $\mathrm{V}, \mathrm{W}, \mathrm{k})$

\% Parameters 


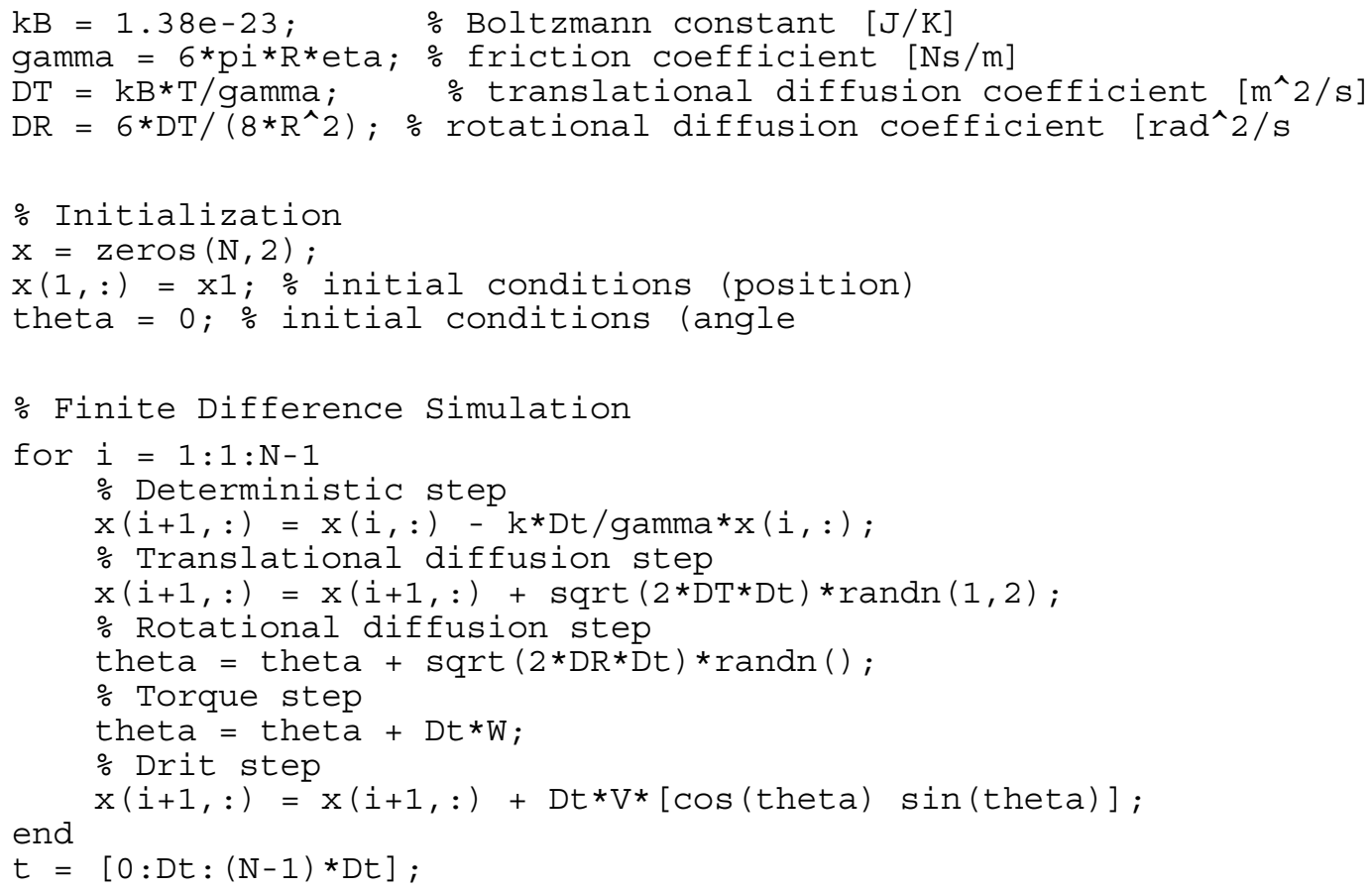

\section{COMPLEX FORCE FIELDS}

The presence of more complex force fields, such as non-conservative force fields [32-38] or random optical potential [39-49], can be taken into account by modeling a generic force in Eq. (1) as explained in Ref. [2].

\section{REFERENCES}

[1] Ashkin, A., "Acceleration and trapping of particles by radiation pressure," Phys. Rev. Lett. 24, 156-159 (1970).

[2] Volpe, G. and Volpe, G., "Simulation of a Brownian particle in an optical trap," Am. J. Phys. 81, 224-230 (2013).

[3] Volpe, G., Gigan, S., and Volpe, G., "Simulation of the active Brownian motion of a microswimmer," Am. J. Phys. 82, 659-664 (2014).

[4] Scilab $<$ www.scilab.org/ $>$.

[5] GNU Octave software, <www.gnu.org/software/octave/>

[6] Ebbens, S.J. and Howse, J.R., "In pursuit of propulsion at the nanoscale," Soft Matter 6, 726-738 (2010).

[7] Poon, W.C.K., "From Clarkia to Escherichia and Janus: The physics of natural and synthetic active colloids," eprint arXiv:1306.4799 (2013).

[8] Berg, H., E. coli in Motion (Springer Verlag, New York, NY, 2004).

[9] Weibel, D.B., Garstecki, P., Ryan, D., DiLuzio, W.R., Mayer, M., Seto, J.E. and Whitesides, G.M., "Microoxen: Microorganisms to move microscale loads," Proc. Natl. Acad. Sci. U.S.A. 102, 11963-11967 (2005).

[10] Ford, R.M. and Harvey, R.W., "Role of chemotaxis in the transport of bacteria through saturated porous media, Adv. Water Res. 30, 1608-1617 (2007).

[11] Yang, W., Misko, V.R., Nelissen, K. Kong, M. and Peeters, F.M., "Using self-driven microswimmers for particle separation," Soft Matter 8, 5175-5179 (2012).

[12] Drocco, J., Reichhardt, C., and Reichhardt, C., "Bidirectional Sorting of Self-Propelled Microswimmers in the Presence of Asymmetric Barriers," Biophys. J. 104, 496 (2013).

[13] Kaiser, A., Popowa, K., Wensink, H. H., and Löwen, H., "Capturing self-propelled particles in a moving micro wedge," Phys. Rev. E 88, 022311 (2013). 
[14]Reichhardt, C., and Olson Reichhardt, C. J., "Dynamics and separation of circularly moving particles in asymmetrically patterned arrays," Phys. Rev. E 88, 042306 (2013).

[15] Speck, T., Bialké, J., Menzel, A. M., and Löwen, H., "Effective Cahn-Hilliard equation for the phase separation of active Brownian particles,” Phys. Rev. Lett. 112, 218304 (2014).

[16]Erdmann, U., Ebeling, W., Schimansky-Geier, L. and Schweitzer, F., "Brownian particles far from equilibrium,” Eur. Phys. J. B: Condens. Matt. Comp. Sys. 15, 105-113 (2000).

[17] Hänggi, P. and Marchesoni, F., "Artificial Brownian motors: Controlling transport on the nanoscale," Rev. Mod. Phys. 81, 387-442 (2009).

[18] Golestanian, R., Liverpool, T.B. and Ajdari, A., "Propulsion of a molecular machine by asymmetric distribution of reaction products," Phys. Rev. Lett. 94, 220801 (2005).

[19] Paxton, W.F., Sen, A. and Mallouk, T.E, "Motility of catalytic nanoparticles through self-generated forces," Chem. Eur. J. 11, 6462-6470 (2005).

[20]Dreyfus, R., Baudry, J., Roper, M.L., Fermigier, M., Stone, H.A. and Bibette, J., "Microscopic artificial swimmers," Nature 437, 862-865 (2005).

[21] Howse, J.R., Jones, R.A.L., Ryan, A.J., Gough, T., Vafabakhsh, R. and Golestanian, R., "Self-motile colloidal particles: From directed propulsion to random walk," Phys. Rev. Lett. 99, 048102 (2007).

[22] Tierno, P., Golestanian, R., Pagonabarraga, I. and Sagués, F., "Magnetically actuated colloidal microswimmers," J. Phys. Chem. B 112, 16525-16528 (2008).

[23] Palacci, J., Cottin-Bizonne, C., Ybert, C. and Bocquet, L., "Sedimentation and effective temperature of active colloidal suspensions," Phys. Rev. Lett. 105, 088304 (2010).

[24] Popescu, M.N., Dietrich, S., Tasinkevych, M. and Ralston, J., "Phoretic motion of spheroidal particles due to self-generated solute gradients," Eur. Phys. J. E 31, 351-367 (2010).

[25] Volpe, G., Buttinoni, I., Vogt, D., Kümmerer, H.-J. and Bechinger, C., "Microswimmers in patterned environments," Soft Matter 7, 8810-8815 (2011).

[26] Búzás, A., Kelemen, L., Mathesz, A., Oroszi, L., Vizsnyiczai, G., Vicsek, T. and Ormos, P., "Light sailboats: Laser driven autonomous microrobots," Appl. Phys. Lett. 101, 041111 (2012).

[27] Buttinoni, I., Volpe, G., Kümmel, F., Volpe, G. and Bechinger, C., "Active Brownian motion tunable by light," J. Phys.: Condens. Matter 24, 284129-1-6 (2012).

[28] Koumakis, N., Lepore, A., Maggi, C. and Di Leonardo, R. "Targeted delivery of colloids by swimming bacteria," Nat. Commun. 4, 2588 (2013).

[29] Van Teeffelen, S. and Löwen, H., "Dynamics of a Brownian circle swimmer," Phys. Rev. E 78, 020101 (2008).

[30] Mijalkov, M. and Volpe, G., "Sorting of Chiral microswimers," Soft Matter 9, 6376-6381 (2013).

[31]Purcell, E. M., "Life at low Reynolds numbers," Am. J. Phys. 45, 3-11 (1977).

[32] Bishop, A. I., Nieminen, T. A., Heckenberg, N. R., and Rubinsztein-Dunlop, H., "Optical application and measurement of torque on microparticles of isotropic nonabsorbing material," Phys. Rev. A 68, 033802 (2003).

[33] La Porta, A., and Wang, M. D., "Optical torque wrench: Angular trapping, rotation, and torque detection of quartz microparticles,” Phys. Rev. Lett. 92, 190801 (2004).

[34] Volpe, G., and Petrov, D., "Torque detection using brownian fluctuations," Phys. Rev. Lett. 97, 210603 (2006).

[35] Volpe, G., Volpe, G., and Petrov, D., "Brownian motion in a nonhomogeneous force field and photonic force microscope," Phys. Rev. E 76, 061118 (2007).

[36] Volpe, G., Volpe, G., and Petrov, D., "Singular-point characterization in microscopic flows," Phys. Rev. E 77, 037301 (2008).

[37]Borghese, F., Denti, P., Saija, R., Iatì, M. A., and Maragó, O. M., "Radiation torque and force on optically trapped linear nanostructures," Phys. Rev. Lett. 100, 163903 (2008).

[38] Pesce, G., Volpe, G., De Luca, A. C., Rusciano, G., and Volpe, G., "Quantitative assessment of nonconservative radiation forces in an optical trap," EPL 86, 38002 (2009).

[39] Goodman, J.W., "Some fundamental properties of speckle," J. Opt. Soc. Am. 66, 11451150 (1976).

[40] Boiron, D., Mennerat-Robilliard, C., Fournier, J.M., Guidoni, L., Salomon, C. and Grynberg, G., "Trapping and cooling cesium atoms in a speckle field," Eur. Phys. J. D 7, 373-377 (1999).

[41] Shvedov, V.G., Rode, A.V., Izdebskaya, Y.V., Leykam, D., Desyatnikov, A.S., Krolikowski, W. and Kivshar, Y.S. "Laser speckle field as a multiple particle trap," J. Opt. 12, 124003 (2010).

[42] Shvedov, V.G., Rode, A.V., Izdebskaya, Y.V., Desyatnikov, A.S., Krolikowski, W. and Kivshar, Y.S., "Selective trapping of multiple particles by volume speckle field," Opt. Express 18, 3137-3142 (2010). 
[43] Staforelli, J.P., Brito, J.M.,Vera, E., Solano, P. and Lencina, A.A., “A clustered speckle approach to optical trapping," Opt. Commun. 283, 4722-4726 (2010).

[44]Hanes, R.D.L., Dalle-Ferrier, C., Schmiedeberg, M., Jenkins, M.C. and Egelhaaf, S.U., "Colloids in one dimensional random energy landscapes," Soft Matter 8, 2714-2723 (2012).

[45]Douglass, K.M. Sukhov, S. and Dogariu, A., "Superdiffusion in optically controlled active media," Nature Photon. 6, 834-837 (2012).

[46] Evers, F., Zunke, C., Hanes, R.D.L., Bewerunge, J., Ladadwa, I., Heuer, A. and Egelhaaf, S.U., "Particle dynamics in two-dimensional random-energy landscapes: Experiments and simulations," Phys. Rev. E 88, 022125 (2013).

[47]Evers, F., Hanes, R.D.L., Zunke, C., Capellmann, R.F., Bewerunge, J., Dalle-Ferrier, C., Jenkins, M.C., Ladadwa, I., Heuer, A., Castañeda-Priego, R. and Egelhaaf, S.U., "Colloids in light fields: Particle dynamics in random and periodic energy landscapes," Eur. Phys.J. Special Topics 222, 2995-3009 (2013).

[48] Volpe, G., Volpe, G. and Gigan, S. "Brownian Motion in a Speckle Light Field: Tunable Anomalous Diffusion and Selective Optical Manipulation," Sci. Rep. 4, 3936 (2014).

[49] Volpe, G., Kurz, L., Callegari, A., Volpe, G. and Gigan, S. "Speckle Optical Tweezers: micromanipulation with random light fields," Opt. Express 22, 18159-18167 (2014). 